\title{
BMJ Open IDEA intervention to prevent depressive symptoms and promote well-being in early-stage dementia: protocol for a randomised controlled feasibility study
}

\author{
Remco Tuijt, Gill Livingston, Rebecca L Gould, Rebecca Jones, \\ Elisabet Sole Verdaguer, Vasiliki Orgeta
}

To cite: Tuijt R, Livingston $\mathrm{G}$ Gould RL, et al. IDEA intervention to prevent depressive symptoms and promote well-being in earlystage dementia: protocol for a randomised controlled feasibility study. BMJ Open 2018;8:e21074. doi:10.1136/ bmjopen-2017-021074

- Prepublication history for this paper is available online. To view these files, please visit the journal online (http://dx.doi org/10.1136/bmjopen-2017021074).

Received 8 December 2017 Accepted 12 December 2017

Check for updates

Division of Psychiatry, University College London, London, UK

Correspondence to

Dr Vasiliki Orgeta;

v.orgeta@ucl.ac.uk

\section{ABSTRACT}

Objective Depressive symptoms are common among people with dementia, impacting quality of life and cognitive and functional decline. Currently, little is known about the acceptability and feasibility of psychological interventions for people with mild dementia, with recent reviews identifying the need for further evidence. Developing and evaluating psychological interventions to prevent and treat these symptoms is, therefore, an important clinical and research priority. This protocol describes a study testing the acceptability and feasibility of a manual-based behavioural activation (BA) intervention for preventing and treating depressive symptoms in people with mild dementia. The aim of this study is to explore the feasibility of conducting a pragmatic multicentre randomised controlled trial of clinical effectiveness of an eight-session intervention. The Intervention to prevent Depressive symptoms and promote well-being in EArlystage dementia (IDEA) programme supports people with dementia and their family carers in identifying and scheduling enjoyable and meaningful activities.

Methods and analysis Sixty people who have received a diagnosis of dementia of any type in the last 6 months will be recruited via memory clinics. Further criteria are a Mini-Mental State Examination score of $\geq 20$, and a family carer who can assist with the intervention. Consenting participants will be randomised in a ratio of 2:1 to BA or to treatment as usual. Analyses will estimate parameters such as rates of recruitment, retention and number of sessions completed. Questionnaires measuring depressive symptoms and quality of life for both the person with dementia and their carer will be completed at baseline, 3 and 6 months. Qualitative interviews will explore acceptability of the intervention, study procedures and experiences of the sessions.

Ethics and dissemination This study received a favourable ethical opinion from the London Camberwell St Giles Research Ethics Committee (16/L0/0540). We will disseminate findings at key conferences, the Alzheimer's Society and University College London websites and local stakeholder events.

Trial registration number ISRCTN75503960; Pre-results.

\section{INTRODUCTION}

Dementia is a leading cause of disability in late life, ${ }^{1}$ with economic costs to society expected to
Strengths and limitations of this study

- This will be the first study to provide acceptability and feasibility data for a psychological intervention based on behavioural activation for preventing and treating depressive symptoms in people with mild dementia.

- Explores feasibility parameters such as rates of recruitment, follow-up retention and number of sessions completed, with several proposed outcome measures tested for suitability of a full-scale trial.

- By using both quantitative and qualitative data, the results will inform a future large-scale randomised controlled trial of clinical effectiveness.

- Limited to follow-ups at 3 and 6 months.

- People with dementia and their carers will be recruited from two London National Health Service sites which may be associated with sample selection bias.

double from $£ 26$ billion per year to $£ 55$ billion in 2040 in the UK alone. ${ }^{2}$ There are currently over 50 million people living with dementia, with numbers of people affected expected to increase to 66 million by $2030 .{ }^{3}{ }^{4}$ People with dementia are at increased risk of experiencing psychological distress such as depression, ${ }^{5} 6$ which is not only distressing but persistent and associated with increasing cognitive and functional decline, ${ }^{78}$ a risk of earlier care-home admission ${ }^{9}$ and reduced life expectancy. ${ }^{10}$ In line with current National Health Service policies and the National Dementia Strategy, ${ }^{11}$ accessing emotional, social and practical support after diagnosis is an important and timely objective for people with dementia, ${ }^{12} 13$ and it is reasonable to assume that people with dementia who additionally experience symptoms of depression will require more resources and support. In a review of experiences of postdiagnostic treatment, psychological care for people with dementia was described as least developed, ${ }^{14}$ indicating that access to psychological support 
remains poor despite an increasing emphasis on supporting people to maintain a sense of well-being.

Current estimates show that up to $50 \%$ of people living with dementia experience depression at least once during the course of the disease. ${ }^{15}$ Major depressive disorder affects approximately $20 \%-30 \%$ of people with dementia, ${ }^{715}$ with personal or family history of depression $^{16}$ and a younger age at onset of dementia increasing the risk of depression. ${ }^{17}$ Subclinical or subthreshold symptoms of depression occur at a higher rate than clinical depression, ${ }^{18}$ tend to be highly persistent ${ }^{7}$ and are often experienced during the early stages of the disease. ${ }^{19}$ Both depression and less severe depressive symptoms are sources of excess disability for people with dementia, therefore both major and subthreshold symptoms of depression are considered clinically important. ${ }^{182} 2$

Although depression is associated with high burden for people with dementia and their carers, there are currently no interventions available to prevent depressive symptoms occurring or becoming worse, which is key given poor efficacy of antidepressants. ${ }^{21}$ Despite limited efficacy of pharmacological interventions and increased risk of side effects, about a third of people with Alzheimer's disease living in the community are prescribed antidepressants, ${ }^{22} 23$ and up to $40 \%$ in care homes ${ }^{24}$ indicating a high need to manage these symptoms. ${ }^{19}$

A Cochrane Review ${ }^{25}$ of the effects of psychological treatments for people with dementia found that these treatments may contribute to a reduction in depressive symptoms, however evidence comes mostly from small-scale studies, with heterogeneous treatments. ${ }^{25} \mathrm{~A}$ recent systematic review of behavioural activation (BA) interventions for older people found that these are associated with a reduction in depressive symptoms in older people without dementia living in the community. ${ }^{26}$ The review also highlighted that most studies so far including people with dementia do not use well-defined interventions. The long-term aim of this research is to test the effectiveness and cost-effectiveness of BA for people with dementia living in the community.

This study aims to establish the acceptability and feasibility of an 8-week intervention using BA principles ${ }^{27} 28$ developed after extensive consultations and field testing with people with mild dementia and their family carers. The study design is a feasibility randomised controlled trial (RCT), with two treatment arms (BA vs treatment as usual) following participants over 8 months. A secondary objective is to test the feasibility of procedures for conducting and planning a multicentre RCT by exploring trial processes, and acceptability and feasibility of the intervention.

\section{METHODS AND ANALYSIS}

\section{Participant recruitment}

\section{Inclusion criteria}

Participants will be people who have received a diagnosis of dementia of any type in the last 6 months and: (1) are living in the community, (2) have a family carer who is available to participate and support the person in the intervention and (3) have mild dementia (determined by a Mini- Mental State Examination score of $\geq 20$ ).

\section{Exclusion criteria}

Participants will be excluded if (1) they are deemed by their clinical team to be at risk of self-harm (excluding neglect) or a risk to others, (2) have difficulties communicating in English or (3) are already taking part in another intervention study. People with previous depression, or previous or current treatment with antidepressants or experience of psychological treatments will not be excluded. The study will recruit a total of 60 people with dementia and their carers from memory clinics and community mental health teams of older people in London. Potential participants will be recruited by clinicians in relevant Trusts, by invitation letter or by a 'consent to contact' approach.

\section{Sample size considerations and randomisation}

A total of 60 people will be recruited ${ }^{29} 30$ and randomised in a ratio of 2:1 to either the $\mathrm{BA}$ intervention or treatment as usual. Among the 40 participants in the BA arm, we will be able to estimate $75 \%$ acceptability of the IDEA intervention with a $95 \%$ CI of $59 \%$ to $87 \%$.

Participant randomisation will be undertaken at University College London (UCL) using a randomisation system stratified by site provided by internet-based sealed envelope codes, based on random permuted blocks of sizes three and six to allow a 2:1 allocation to intervention and treatment as usual. Randomisation allocation will be sent by automated email, to the non-blind researcher performing the randomisation using their unique user name and password.

\section{Treatment as usual}

This study is designed to be a pragmatic feasibility study, and no participants will be denied access to any treatment that they would have had access to. Both groups will receive regular treatment as usual, which is expected to be in line with the National Institute for Health and Care Excellence guidelines for treatment of dementia, details of which will be recorded for each participant using the Client Service Receipt Inventory (CSRI) ${ }^{31}$

\section{Blinding}

All follow-up assessments will be conducted by a researcher who is blind to treatment allocation. In trials of psychological interventions, it is difficult to blind therapists and participants to which intervention they are delivering or receiving. Any incidents of non-blinding will be recorded to inform the future trial.

\section{Intervention}

The proposed intervention will follow theory and models of $\mathrm{BA},{ }^{27} 28$ and key components identified to be associated with effectiveness in trials of older people. ${ }^{26} \mathrm{BA}$ is defined as a brief structured therapeutic approach aimed at increasing engagement in adaptive activities often 
associated with pleasure and mastery, through structured activity scheduling and monitoring of mood, supplemented by additional behavioural strategies, such as relaxation techniques, and hierarchical construction of goals. ${ }^{26}$

The IDEA intervention will include:

a. identifying pleasant activities for the person and developing and agreeing a plan of which activities to implement

b. setting goals about implementing these activities

c. monitoring and reviewing activities on a weekly basis

d. teaching of specific relaxation skills ${ }^{32}$

e. discussing accessing help

f. making a plan for the future.

The intervention will be an individual home-based manualised programme, comprised of a total of eight weekly 1-hour sessions, delivered by a psychology graduate who will be trained in the intervention and supervised monthly by one of the coinvestigators. Based on the suggestions and observations when developing the intervention, session frequency will depend on participants, with sessions completed over 8-12 weeks.

\section{Adherence to treatment protocol}

A manual guiding therapists in delivering the intervention will be developed, describing its key components. The feasibility of the treatment-adherence measures and competency assessment will be assessed by developing a checklist for use in the main trial.

\section{Subsequent assessments of outcomes}

All participants receiving the intervention will be asked to complete a follow-up qualitative interview at their home administered by a researcher not providing the intervention. These interviews will examine experiences and expectations of the intervention, and suitability of intervention materials in order to inform their further refinement in the main trial. Participants will be recruited until theoretical saturation has been achieved. Data on acceptability, completion rates and attrition will be supplemented by qualitative data.

\section{Proposed outcome measures for the full-scale clinical trial}

The flow diagram of the current trial is presented in figure 1. In order to inform the future trial, outcome measures will be trialled for suitability. Depressive symptoms is proposed as the outcome for the main study. Carers will complete generic health and quality of life measures, and resource use questionnaires to examine the feasibility of cost-effectiveness analysis. Questionnaires will be completed at baseline, 3 and 6 months after randomisation.

\section{Outcomes tested for acceptability for the main trial}

1. Depressive symptoms-Cornell Scale for Depression in Dementia (CSDD) ${ }^{33}$ The CSDD is a 19-item interviewer-administered measure which uses information provided by interviewing the person with dementia and their carer. Symptoms are described to the carer as they appear on the scale. Where there is a discrepancy between the carer and the researcher's ratings the carer is reinterviewed before making a final judgement.

2. Self-rated and carer-rated dementia-specific quality of life for the person with dementia-DEMQOL andDEMQOL-proxy. ${ }^{34}$ The DEMQOL measures quality of life,${ }^{35}$ in five domains, including daily activities, health and well-being, cognitive functioning, social relationships and self-concept. The scale is used as a self-rated or carer-rated report, administered to the person with dementia and carer, with established validity. ${ }^{36}$

3. Self-rated and carer-rated quality of life using the European Quality of Life-5 Dimensions (EQ-5D) for the person with dementia, measured using the three-level response version of the EQ-5D, a standardised instrument for use as a measure of health outcome. ${ }^{37}$

4. Neuropsychiatric symptoms-Neuropsychiatric Inventory (NPI). ${ }^{38}$ The NPI assesses 12 behavioural disturbances occurring in people with dementia as rated by the carer, using a screening strategy to minimise administration time by examining and scoring only those behavioural domains that have positive responses to screening questions. Both frequency and severity of each behaviour are also rated with higher scores indicative of more symptoms. Both validity and reliability of the measure have been established. ${ }^{35}$

5. Health services utilisation-CSRI. ${ }^{39}$ The CSRI will be used to collect feasibility data on the use of health and social care services provided by public or non-public bodies, and information on carers' costs and participants' use of health and social care services to inform the full trial. The feasibility of collecting service-use data will be assessed, which will allow identification of services received in both groups and any changes that may occur.

6. Carers' mental health-Hospital and Anxiety Depression Scale (HADS). ${ }^{40}$ Depressive and anxiety symptoms in carers will be measured using HADS, a self-rated measure, generating scores for both generalised anxiety and depressive symptoms, used widely to identify caseness for clinically significant depression and anxiety. ${ }^{41}$

7. Carers' quality of life-the EQ-5D $\mathrm{D}^{37}$ and Short Form12 Health Survey (SF-12). ${ }^{42}$ Carer health-related quality of life will be measured using the EQ-5D. Carers' mental and physical health will be measured by the SF-12, measuring health by standardised responses, expressed in terms of two meta-scores: the physical component summary and the mental component summary.

\section{Safety monitoring}

Adverse events will be closely monitored. These are events that are likely to affect to a significant degree the safety or physical or mental integrity of the participants in the trial. The sponsor will be notified immediately of any case where the above definition applies during the trial. 


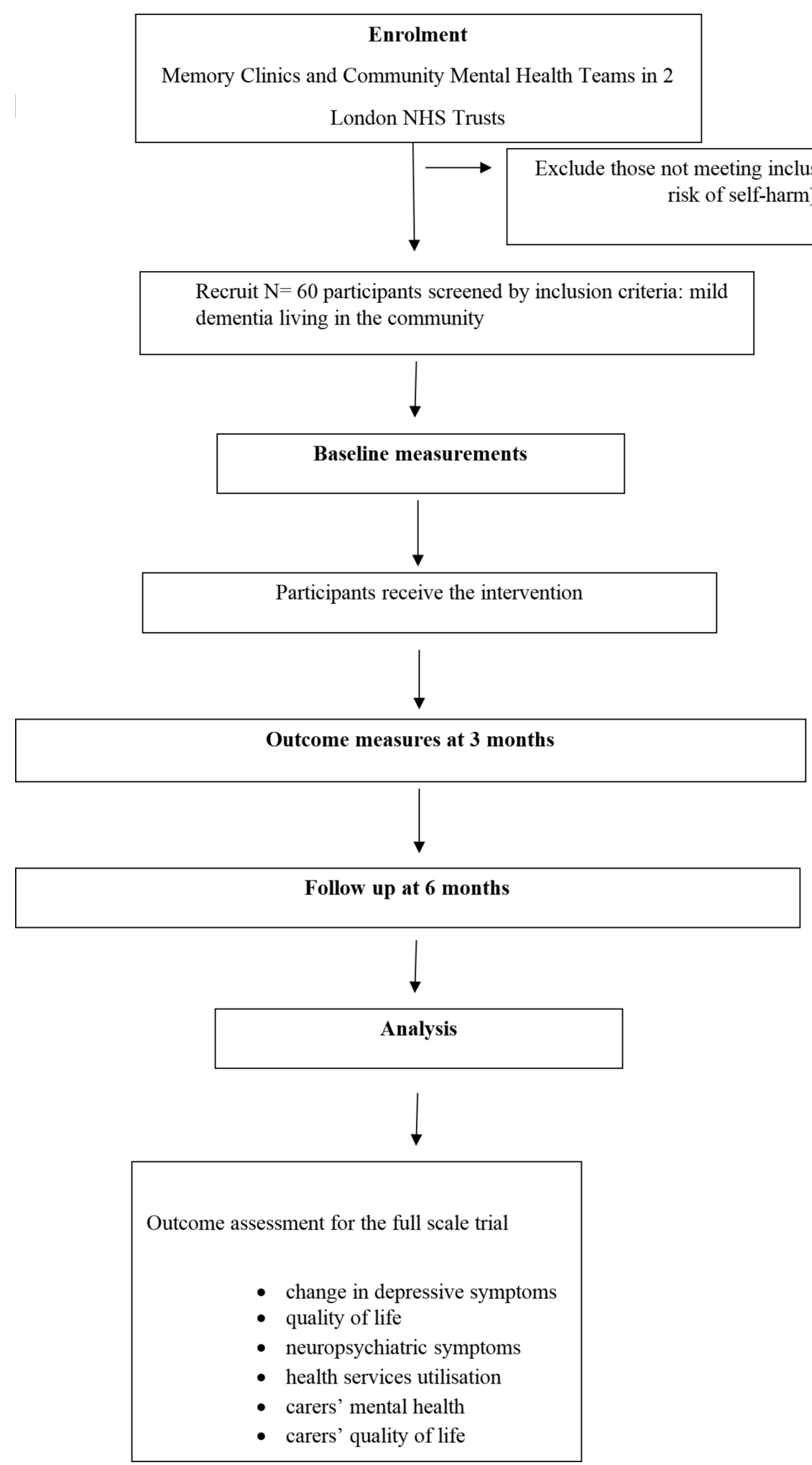

Figure 1 Flow diagram. NHS, National Health Service.

\section{Statistical analysis plan}

Given this is a feasibility study, it does not aim to provide a hypothesis test of the effectiveness of the intervention but to estimate feasibility study parameters. The main aims, therefore, are feasibility of the randomised controlled study and acceptability of the intervention for people with dementia and their carers. We will, therefore, test analysis procedures in order to inform the statistical analysis plan of the main trial as opposed to making statistical comparisons of outcomes between the intervention and treatment as usual groups. The analyses will include the following:

- rates of clinicians' referrals, number of participants recruited and randomised to the study (number of 
dyads referred and recruited per month, percentage reaching inclusion criteria and any barriers or facilitators to recruitment);

- percentage of eligible participants who consent to the study;

- percentage of BA sessions completed (percentage in the BA group completing all sessions);

- follow-up rates and number completing each outcome measure proposed for the main trial.

We will produce a Consolidated Standards of Reporting Trials diagram to represent numbers of people with dementia eligible for inclusion, numbers recruited, randomised and completing the study. Details of participants who meet the inclusion criteria but are not randomised will be recorded in order to inform the future trial. Essential baseline information will be recorded which will include quantitative and qualitative information (ie, specifics of diagnosis, psychiatric history, use of medications and demographics). In line with the current recommendations of Good Clinical Practice, analyses will be descriptive. We will estimate SDs (95\% CIs) of potential outcome measures at baseline, 3 and 6 months and changes in scores from baseline. We will use rates of missing questionnaire data to inform the full trial. For the feasibility economic evaluation component, we will collect data on resource use and costs for the future trial including resources required to deliver the intervention.

\section{ETHICS AND DISSEMINATION Ethics}

The study is registered as a clinical trial and has been allocated an International Standard Randomised Controlled Trial Number (ISRCTN75503960). As the intervention is a psychological therapy, the trial is not covered by the Medicines for Human Use (Clinical Trials) Regulations 2004.

\section{Informed consent and withdrawal from the study}

Participants will be in the mild stages of dementia, and therefore would generally be expected to be able to provide informed consent. In instances where the participant's level of impairment increases, such that they are no longer able to provide informed consent, the provisions of the Mental Capacity Act will be followed. Participants will be informed of their right to withdraw at any time without their care being affected in any way.

\section{Data management}

All participant information will be stored in accordance with the UK Data protection Act 1998 guidance, with all personally identifiable information stored in locked cabinets and stored separately from study data which will be anonymised and saved on password-protected computers at UCL, in line with UCL Data Protection Policy.

\section{Patient and public involvement}

Local user groups of people with dementia and their carers, and professionals supporting them have been consulted and involved in the design of the study, the development of the intervention and recruitment strategies. ${ }^{43}$ A dissemination strategy will be developed that will identify key stakeholder groups who will be communicated of the study's findings. We will also consult our Patient and Public Involvement members for the design of the main trial.

\section{Oversight committees}

A Trial Steering Committee has been set-up and will include an independent chair, two independent members and the study's coinvestigator(s).

\section{DISCUSSION}

This study will evaluate the acceptability and feasibility of a psychological intervention using BA principles to prevent and treat depressive symptoms for people with mild dementia informing a clinical and cost-effectiveness trial. In line with Medical Research Council guidelines ${ }^{44}$ we will use data from this study to further refine the intervention, produce a training package and inform approaches for recruitment and analyses for the full-scale trial. This feasibility study is necessary preparatory work to inform a full RCT and the psychological care of people with dementia.

\section{TRIAL SPONSORSHIP}

UCL is the nominated sponsor.

\section{DATE OF STUDY}

08 of January 2016 to 08 of January 2019.

Contributors V0 and GL developed the original concept of the trial, and VO drafted the original protocol; RLG provided guidance on the development of the intervention; RJ provided statistical input; RT adapted the trial proposal as a protocol paper with help from ESV; VO, RT, RLG and GL have contributed to the development of the IDEA intervention. All authors reviewed and commented on drafts of the protocol and paper. All authors read and approved the final manuscript.

Funding This work is funded by Alzheimer's Society protocol number 15/0910. Grant number is 259 (AS-SF-15-005).

Competing interests None declared.

Patient consent Not required.

Ethics approval The study has received a favourable ethical opinion from the London Camberwell St Giles Research Ethics Committee (16/L0/0540).

Provenance and peer review Not commissioned; peer reviewed for ethical and funding approval prior to submission.

Open Access This is an Open Access article distributed in accordance with the Creative Commons Attribution Non Commercial (CC BY-NC 4.0) license, which permits others to distribute, remix, adapt, build upon this work non-commercially, and license their derivative works on different terms, provided the original work is properly cited and the use is non-commercial. See: http://creativecommons.org/ licenses/by-nc/4.0/

(c) Article author(s) (or their employer(s) unless otherwise stated in the text of the article) 2018. All rights reserved. No commercial use is permitted unless otherwise expressly granted. 


\section{REFERENCES}

1. Prince M, Bryce R, Albanese E, et al. The global prevalence of dementia: a systematic review and metaanalysis. Alzheimers Dement 2013;9:63-75.

2. Prince M, Knapp P, Guerchet M, et al. Dementia UK: an update. London: Alzheimer's Society, 2014.

3. Rocca WA, Petersen RC, Knopman DS, et al. Trends in the incidence and prevalence of Alzheimer's disease, dementia, and cognitive impairment in the United States. Alzheimers Dement 2011;7:80-93.

4. Matthews FE, Arthur A, Barnes LE, et al. A two-decade comparison of prevalence of dementia in individuals aged 65 years and older from three geographical areas of England: results of the cognitive function and ageing study I and II. Lancet 2013;382:1405-12.

5. Weiner MF, Doody RS, Sairam R, et al. Prevalence and incidence of major depressive disorder in Alzheimer's disease: findings from two databases. Dement Geriatr Cogn Disord 2002;13:8-12.

6. Enache D, Winblad B, Aarsland D. Depression in dementia: epidemiology, mechanisms, and treatment. Curr Opin Psychiatry 2011;24:461-72.

7. Ballard C, Bannister C, Solis M, et al. The prevalence, associations and symptoms of depression amongst dementia sufferers. J Affect Disord 1996;36:135-44.

8. Fritze F, Ehrt U, Hortobagyi T, et al. Depressive symptoms in Alzheimer's disease and lewy body dementia: a one-year follow-up study. Dement Geriatr Cogn Disord 2011;32:143-9.

9. Gaugler JE, Yu F, Krichbaum K, et al. Predictors of nursing home admission for persons with dementia. Med Care 2009;47:191-8.

10. Burns $A$, Lewis $G$, Jacoby $R$, et al. Factors affecting survival in Alzheimer's disease. Psychol Med 1991;21:363-70.

11. Burns A, lliffe S. Dementia. BMJ 2009;338:b75.

12. von Kutzleben M, Schmid W, Halek M, et al. Community-dwelling persons with dementia: what do they need? What do they demand? What do they do? A systematic review on the subjective experiences of persons with dementia. Aging Ment Health 2012;16:378-90.

13. Miranda-Castillo $C$, Woods B, Orrell M. The needs of people with dementia living at home from user, caregiver and professional perspectives: a cross-sectional survey. BMC Health Serv Res 2013;13:43,6963-13-43.

14. Bunn F, Goodman C, Sworn K, et al. Psychosocial factors that shape patient and carer experiences of dementia diagnosis and treatment: a systematic review of qualitative studies. PLoS Med 2012;9:e1001331.

15. Starkstein SE, Jorge R, Mizrahi R, et al. The construct of minor and major depression in Alzheimer's disease. Am J Psychiatry 2005;162:2086-93.

16. Strauss ME, Ogrocki PK. Confirmation of an association between family history of affective disorder and the depressive syndrome in Alzheimer's disease. Am J Psychiatry 1996;153:1340-2.

17. Butt ZA, Strauss ME. Relationship of family and personal history to the occurrence of depression in persons with Alzheimer's disease. Am J Geriatr Psychiatry 2001;9:249-54.

18. Migliorelli R, Tesón A, Sabe L, et al. Prevalence and correlates of dysthymia and major depression among patients with Alzheimer's disease. Am J Psychiatry 1995;152:37-44.

19. Holtzer R, Scarmeas N, Wegesin DJ, et al. Depressive symptoms in Alzheimer's disease: natural course and temporal relation to function and cognitive status. J Am Geriatr Soc 2005;53:2083-9.

20. Reichman WE, Coyne AC. Depressive symptoms in Alzheimer's disease and multi-infarct dementia. J Geriatr Psychiatry Neurol 1995;8:96-9.

21. Orgeta V, Tabet N, Nilforooshan R, et al. Efficacy of Antidepressants for Depression in Alzheimer's Disease: Systematic Review and MetaAnalysis. J Alzheimers Dis 2017;58:725-33.

22. Laitinen ML, Lönnroos E, Bell JS, et al. Use of antidepressants among community-dwelling persons with Alzheimer's disease: a nationwide register-based study. Int Psychogeriatr 2015;27:1-4.
23. Arbus C, Gardette V, Bui E, et al. Antidepressant use in Alzheimer's disease patients: results of the REAL.FR cohort. Int Psychogeriatr 2010;22:120-8.

24. Livingston G, Barber J, Marston L, et al. Prevalence of and associations with agitation in residents with dementia living in care homes: MARQUE cross-sectional study. BJPsych Open 2017;3:171-8.

25. Orgeta V, Qazi A, Spector A, et al. Psychological treatments for depression and anxiety in dementia and mild cognitive impairment: systematic review and meta-analysis. Br J Psychiatry 2015;207:293-8.

26. Orgeta V, Brede J, Livingston G. Behavioural activation for depression in older people: systematic review and meta-analysis. $\mathrm{Br}$ J Psychiatry 2017;211:274-9.

27. Lewinsohn PM, Muñoz RF, Youngren MA, et al. Control your depression. New York: Prentice Hall Press, 1986.

28. Dimidjian S, Barrera M, Martell C, et al. The origins and current status of behavioral activation treatments for depression. Annu Rev Clin Psychol 2011;7:1-38.

29. Sim J, Lewis M. The size of a pilot study for a clinical trial should be calculated in relation to considerations of precision and efficiency. J Clin Epidemiol 2012;65:301-8.

30. Julious SA. Sample size of 12 per group rule of thumb for a pilot study. Pharm Stat 2005;4:287-91.

31. Beecham J, Knapp M. Costing psychiatric interventions. In: Thornicroft G, ed. Measuring mental health needs: Gaskell, 2001:200-24.

32. Colom F. Keeping therapies simple: psychoeducation in the prevention of relapse in affective disorders. $\mathrm{Br} J$ Psychiatry 2011;198:338-40.

33. Alexopoulos GS, Abrams RC, Young RC, et al. Cornell scale for depression in dementia. Biol Psychiatry 1988;23:271-84.

34. Smith SC, Lamping DL, Banerjee S, et al. Measurement of healthrelated quality of life for people with dementia: development of a new instrument (DEMQOL) and an evaluation of current methodology. Health Technol Assess 2005;9:1,93, iii-iv.

35. Perrault A, Oremus M, Demers L, et al. Review of outcome measurement instruments in Alzheimer's disease drug trials: psychometric properties of behavior and mood scales. J Geriatr Psychiatry Neurol 2000;13:181-96.

36. Chua KC, Brown A, Little R, et al. Quality-of-life assessment in dementia: the use of DEMQOL and DEMQOL-Proxy total scores. Qual Life Res 2016;25:3107-18.

37. EuroQol Group. EuroQol-a new facility for the measurement of health-related quality of life. Health Policy 1990;16:199-208.

38. Cummings JL, Mega M, Gray K, et al. The Neuropsychiatric inventory: comprehensive assessment of psychopathology in dementia. Neurology 1994;44:2308-14.

39. Beecham J, Knapp M. Costing psychiatric interventions. In: Thornicroft G, Brewin C, Wing J, eds. Measuring mental health needs. London, 1992:163-83.

40. Zigmond AS, Snaith RP. The hospital anxiety and depression scale. Acta Psychiatr Scand 1983;67:361-70.

41. Mykletun A, Stordal E, Dahl AA. Hospital Anxiety and Depression (HAD) scale: factor structure, item analyses and internal consistency in a large population. Br J Psychiatry 2001;179:540-4.

42. Ware J, Kosinski M, Keller SD. A 12-Item short-form health survey: construction of scales and preliminary tests of reliability and validity. Med Care 1996;34:220-33.

43. INVOLVE. Briefing notes for researchers: involving the public in NHS, public health and social care research. Eastleigh: INVOLVE, 2010.

44. Craig P, Dieppe P, Macintyre S, et al. Developing and evaluating complex interventions: the new medical research council guidance. Int J Nurs Stud 2013;50:587-92. 